\section{Robert J. Cava selected as MRS Medalist for work on 3D topological insulators}

$\mathrm{R}$ obert J. Cava, Department of Chemistry, Princeton University, has received the 2016 Materials Research Society (MRS) Medal. He is cited for his "pioneering contributions in the discovery of new classes of 3D topological insulators." Cava will be recognized during the Award Ceremony at the 2016 MRS Fall Meeting in Boston.

Cava has worked in combining physics and chemistry to create accessible materials that can be widely studied through a process of understanding the physics and how to embody the materials. Although topological surface states were first found on the edges of thin buried layers of $\mathrm{HgTe}$ in quantum wells fabricated by molecular beam epitaxy, their discovery soon thereafter on the surfaces of bulk crystals of Sb-doped Bi grown by Cava caused a rise of interest and activity in the field.

Theorists at the University of Pennsylvania had predicted the existence of the exotic states on the surface of crystals of Sb-doped Bi. Once observed, however, the states turned out to be very complex and were therefore not satisfactory for in-depth study. Physicists asked Cava to think of a not-predicted compound that might have better surface states based on what he understood about the materials science of Sb-doped Bi. He proposed, and then grew crystals and proved, with experimental physicist collaborators, that $\mathrm{Bi}_{2} \mathrm{Se}_{3}$ would be a better host for topological surface states than $\mathrm{Sb}$-doped $\mathrm{Bi} . \mathrm{Bi}_{2} \mathrm{Se}_{3}$ is now considered the "hydrogen atom of topological insulators" because it is an excellent prototype for testing ideas and theories in this field.

Cava continued to make contributions to address questions about the physics of topological surface states by translating those questions into materials terms, and then finding new compounds or altering known compounds. Cava has collaborated extensively with physicists to understand the physics they are trying to probe in topological insulators, and through physics-materials feedback, develop model compounds in singlecrystal form.

Through interactions with experimental and theoretical physicists, Cava learned of a new kind of topological insulator where surface states are present due to the protective character of the surface symmetry (the so-called "topological crystalline insulators") and has grown crystals of those compounds that show the existence of the surface states in $\mathrm{Pb}_{1-x} \mathrm{Sn}_{x} \mathrm{Te}$.

Cava has more than 690 publications and 15 patents. His recognitions include the Linus Pauling Award (ACS), Stephanie Kwolek Award in Materials Chemistry (RSC), James C. McGroddy Prize in New Materials (APS), and the Humboldt Research Award. He is a Fellow of the Neutron Scattering Society of America, The American Ceramic Society, and the American Physical Society. His research emphasizes the relationships between chemistry, crystal structure, and electronic and magnetic properties of non-molecular solids; synthesis, structure, and physical property characterization of new transition-metal oxides, chalcogenides, intermetallics, and pnictides; and the study of the properties and materials chemistry of superconductors, magnetic materials, transparent electronic conductors, dielectrics, thermoelectrics, topological insulators, geometrically frustrated magnets, and correlated electron systems.

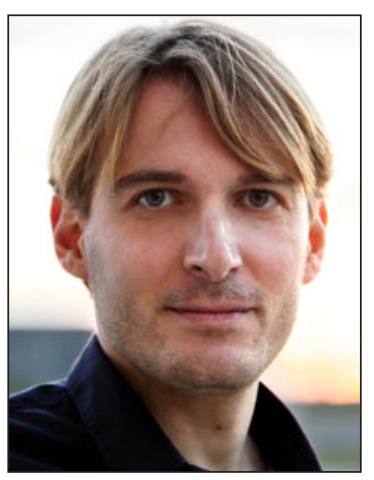

Andrea Alù to present Kavli Foundation Early Career Lectureship in Materials Science

\footnotetext{
$A$ ndrea Alù is the Temple Foundation Endowed Professor in the Department of Electrical and Computer
}

Engineering at The University of Texas at Austin. He will be presenting the Kavli Foundation Early Career Lectureship in
Materials Science on Thursday, December 1, at the 2016 Materials Research Society (MRS) Fall Meeting in Boston.

Alù is a leader in the areas of nanooptics, metamaterials, and plasmonics. $\mathrm{He}$ and his group have introduced the concept of cloaking and low-scattering objects and sensors using metasurfaces. They have also presented groundbreaking advances in the realization and use of nanoclusters and metamaterials to realize optical nanocircuits and nanoantennas, ultrathin broadband circular polarizers, and enhanced optical magnetic response. Alù has also 